\section{The Role of Surface Oxygen in the Growth of Large Single-Crystal Graphene on Copper}

\author{
Yufeng Hao, ${ }^{1}$ M. S. Bharathi, ${ }^{2}$ Lei Wang, ${ }^{3}$ Yuanyue Liu, ${ }^{4}$ Hua Chen, ${ }^{5}$ \\ Shu Nie, ${ }^{6}$ Xiaohan Wang, ${ }^{1}$ Harry Chou, ${ }^{1}$ Cheng Tan, ${ }^{1}$ Babak \\ Fallahazad, ${ }^{7}$ H. Ramanarayan, ${ }^{2}$ Carl W. Magnuson, ${ }^{1}$ Emanuel \\ Tutuc, ${ }^{7}$ Boris I. Yakobson, ${ }^{4}$ Kevin F. McCarty, ${ }^{6}$ Yong-Wei Zhang, ${ }^{2}$ \\ Philip Kim, ${ }^{8}$ James Hone, ${ }^{3}$ Luigi Colombo, ${ }^{9 *}$ Rodney S. Ruoff ${ }^{*}$
}

${ }^{1}$ Department of Mechanical Engineering and the Materials Science and Engineering Program, University of Texas at Austin, Austin, TX 78712, USA. ${ }^{2}$ Institute of High Performance Computing, A*STAR, 138632, Singapore. ${ }^{3}$ Department of Mechanical Engineering, Columbia University, New York, NY 10027, USA. ${ }^{4}$ Department of Mechanical Engineering and Materials Science, and Department of Chemistry, Rice University, Houston, TX 77005, USA. ${ }^{5}$ Department of Physics, University of Texas at Austin, Austin, TX 78712, USA. ${ }^{6}$ Sandia National Laboratories, Livermore, CA 94550, USA. ${ }^{7}$ Microelectronics Research Center, University of Texas at Austin, Austin, TX 78758, USA. ${ }^{8}$ Department of Physics, Columbia University, New York, NY 10027, USA. ${ }^{9}$ Texas Instruments Incorporated, Dallas, TX 75243, USA.

*Corresponding author. E-mail: r.ruoff@mail.utexas.edu (R.S.R); colombo@ti.com (L.C.)

The growth of high-quality single crystals of graphene by chemical vapor deposition on copper $(\mathrm{Cu})$ has not always achieved control over domain size and morphology, and the results vary from lab to lab under presumably similar growth conditions. We discovered that oxygen on the $\mathrm{Cu}$ surface substantially decreased the graphene nucleation density by passivating $\mathrm{Cu}$ surface active sites. Control of surface oxygen enabled repeatable growth of centimeter-scale single-crystal graphene domains. Oxygen also accelerated graphene domain growth and shifted the growth kinetics from edge-attachment-limited to diffusion-limited. Correspondingly, the compact graphene domain shapes became dendritic. The electrical quality of the graphene films was equivalent to mechanically exfoliated graphene, in spite of being grown in the presence of oxygen.

Control of nucleation and growth of graphene during the chemical vapor deposition (CVD) process is important to achieve large, high-quality single crystals $(1-5)$. Much attention has been paid to the process details with emphasis on the parameters of carbon (C) precursors, hydrogen $(\mathrm{H}), \mathrm{Cu}$, temperature, and pressure (6). As such, tuning the $\mathrm{C}: \mathrm{H}$ ratio (6), changing the hydrocarbon and $\mathrm{H}_{2}$ gas pressures (7), and smoothing the $\mathrm{Cu}$ surface prior to growth $(8,9)$, have been used to grow graphene with desirable quality. However, the wide variation in domain size, shape, and film quality from lab to lab suggests that crucial growth parameters still remain unknown or uncontrolled. We show that oxygen on the $\mathrm{Cu}$ surface not only suppresses graphene nucleation, fostering growth of ultra-large single-crystal graphene domains, but also lowers the carbon species edge attachment barrier and shifts the graphene domain shapes from compact to dendritic (10). First-principles calculations and phase field simulations provided a deeper insight into the proposed growth mechanism and reproduced the observed domain shapes.

Oxygen impurities were found to exist at different concentrations across commercially available $\mathrm{Cu}$ foils. Time-of-flight secondary ion mass spectrometry (TOF-SIMS) depth profile results [fig. S2B, see supplementary materials (11)] show that, for two different types of $\mathrm{Cu}$ foils, the oxygen concentrations are $\sim 10^{-2}$ and $\sim 10^{-6}$ at.\% (the latter approaching the detection limit), hereafter referred to as "oxygen-rich $\mathrm{Cu}$ " (OR$\mathrm{Cu}$ ) and 'oxygen-free $\mathrm{Cu}$ ' (OF-Cu), respectively. When the two types of $\mathrm{Cu}$ were used to grow graphene under the same conditions in low pressure CVD, the domain density for OR-Cu is $\sim 0.9 \mathrm{~mm}^{-2}$, more than three orders of magnitude lower than that for $\mathrm{OF}-\mathrm{Cu}$, which is about $2 \times 10^{3} \mathrm{~mm}^{-2}$ (Fig. 1, A and B). We also observed that graphene domains on $\mathrm{OR}-\mathrm{Cu}$ always exhibit dendritic growth fronts, i.e., multi-branched and rough domain edges (Fig. 1A, inset), whereas graphene domains on $\mathrm{OF}-\mathrm{Cu}$ are compact with sharp edges (Fig. 1B, inset). When we exposed $\mathrm{OF}-\mathrm{Cu}$ to $\mathrm{O}_{2}\left(P_{\mathrm{O} 2}=1 \times\right.$ $10^{-3}$ Torr, the substrates are defined as "OF-Cu (O)" hereafter) for $1 \mathrm{~min}$ before introducing methane $\left(\mathrm{CH}_{4}, P_{\mathrm{CH} 4}=1\right.$

$10^{-3}$ Torr), the resulting graphene growth yields a low density of nuclei, $\sim 6 \mathrm{~mm}^{-2}$, and dendritic growth fronts (Fig. 1C) similar to that on OR-Cu. In addition, TOF-SIMS results (fig. S2A) show the presence of surface oxygen after $\mathrm{O}_{2}$ exposure and annealing in $\mathrm{H}_{2}$ $\left(P_{\mathrm{H} 2}=0.1\right.$ Torr $)$. Because graphene growth on $\mathrm{Cu}$ is a surface-mediated process (12), it is reasonable that surface oxygen species, either segregated out of $\mathrm{Cu}$ bulk or adsorbed from $\mathrm{O}_{2}$ exposure, participate in surface reactions and thus responsible for the domain growth characteristics. Both experimental $(13,14)$ and theoretical studies (15) have established that metal surface imperfections, e.g., step edges, defects, impurities, etc., can be active sites for graphene nucleation because of higher $d$-band centers at these lowercoordination sites, which lead to strong binding to adsorbates $(16,17)$. For the same reasons, these active sites are also sinks for oxygen. Thus, surface oxygen on the $\mathrm{Cu}$, regardless of its source, effectively passivated the surface active sites where hydrocarbon accumulation would otherwise have taken place.

In order to further suppress graphene nucleation on $\mathrm{Cu}$, we exposed the $\mathrm{Cu}$ substrates to varying amounts of $\mathrm{O}_{2}\left(P_{\mathrm{O} 2}=1 \times 10^{-3}\right.$ Torr $)$ by simply increasing the exposure time up to $5 \mathrm{~min}$ before introducing $\mathrm{CH}_{4}$. Typically, 2 min of $\mathrm{O}_{2}$ exposure on $\mathrm{OR}-\mathrm{Cu}$ can decrease the nucleation density to $\sim 0.03 \mathrm{~mm}^{-2}$ (Fig. 2A). With longer $\mathrm{O}_{2}$ exposure $(5 \mathrm{~min}$ ), the graphene nucleation density was as low as $\sim 0.01 \mathrm{~mm}^{-2}$, and individual domains grew to a diameter larger than $1 \mathrm{~cm}$ after a 12 hour growth at $P_{\mathrm{CH} 4}=1 \times 10^{-3}$ Torr (Fig. 2B). The same experiments with $\mathrm{OF}-\mathrm{Cu}$ give rise to a similar trend, i.e., the nucleation density decreased with increasing $\mathrm{O}_{2}$ exposure time (Fig. 2D). Oxygen exposure provided a convenient tuning parameter for suppressing graphene nucleation and growing large domains. We used electron backscatter diffraction (EBSD) to map the crystalline orientations of $\mathrm{Cu}$ grains under a graphene domain. The results show that large graphene domains normally grow across several $\mathrm{Cu}$ grains which usually have grain size smaller than a few millimeters even after annealing (Fig. 2C). Low energy electron diffraction (LEED) patterns (fig. S8) taken at different positions on the same domain show that, even though the underlying $\mathrm{Cu}$ is multi-grain, all of the diffraction patterns are aligned with each other, indicating a single-crystal graphene domain. LEED measurements were also performed on other randomly selected large graphene domains with varying shapes (such as in Fig. 2B) on multi-grain $\mathrm{Cu}$, and confirmed that the domains are single crys- 
tals. These observations suggest that single-crystal $\mathrm{Cu}$ substrates are unnecessary to grow large single-crystal graphene films. In addition, we found that high growth temperatures and low $P_{\mathrm{CH} 4}$ facilitated singlecrystal graphene growth. Raman spectra of the domains transferred onto $\mathrm{Si}$ substrates confirmed that they are single-layer with no detectable defect-related D band (fig. S5).

Electrical and magneto-transport measurements were then performed on silicon (fig. S11) and hexagonal boron nitride (h-BN) substrates. Resistivity as a function of back gate voltage of graphene films on h-BN (Fig. 2E) shows narrow and symmetric Dirac peaks with the charge neutrality point $V_{g}=-1.0 \mathrm{~V}$. The carrier mobility measured for three different samples ranges from 40,000 to $65,000 \mathrm{~cm}^{2} \mathrm{~V}^{-1} \mathrm{~s}^{-1}$ at $1.7 \mathrm{~K}$ and from 15,000 to $30,000 \mathrm{~cm}^{2} \mathrm{~V}^{-1} \mathrm{~s}^{-1}$ at room temperature using a carrier density independent fitting method (18). Magneto-transport measurements show that broken symmetry of the four-fold Landau level degeneracy and filling factors of 1-12 were observed under a magnetic field of $8 \mathrm{~T}$ (Fig. 2F). The onset of Shubnikov-de Haas oscillations was also observed at fields below $500 \mathrm{mT}$ (fig. S12). These features suggest that the electrical quality of large graphene domains, despite having been grown with oxygen on $\mathrm{Cu}$, is among the best reported for CVD graphene (19) and comparable to that of micromechanically exfoliated graphene (20).

In addition to decreasing graphene nucleation density, oxygen affected graphene growth kinetics. Figure 3A shows that graphene domains on OF-Cu are compact with a domain size $\sim 15 \mu \mathrm{m}$ after a $32 \mathrm{~min}$ growth at $1035^{\circ} \mathrm{C}$ and $P_{\mathrm{CH} 4}=2 \times 10^{-3}$ Torr. However, when graphene was grown on $\mathrm{OF}-\mathrm{Cu}(\mathrm{O})\left(P_{\mathrm{O} 2}=1 \times 10^{-3}\right.$ Torr, $30 \mathrm{~s}$ exposure $)$ under the same conditions and for the same growth time, the domain size increased to $\sim 100 \mu \mathrm{m}$ (Fig. 3B). This acceleration of graphene domain growth by surface oxygen may seem counterintuitive because oxygen has been associated with carbon species oxidation and graphene etching (21). Oxygen also decreased the graphene film coverage on the $\mathrm{Cu}$ substrates by 5-10 times after $32 \mathrm{~min}$ growth (11). This decrease resulted from the nucleation density on $\mathrm{OF}-\mathrm{Cu}(\mathrm{O})$ being more than two orders of magnitude lower despite the higher individual domain growth rate (11).

We visualize the time-evolution of domain growth at different growth temperatures with carbon isotope labeling and Raman mapping (Fig. 3, $\mathrm{C}$ to $\mathrm{F}$, and fig. S7). Over a wide temperature range, the domains on OF$\mathrm{Cu}$ remained compact hexagons as they grew; whereas on $\mathrm{OF}-\mathrm{Cu}(\mathrm{O})$ the domains were always multi-branched and dendritic. The consistent domain shapes suggest that the kinetics do not change throughout the growth. Also, the radial growth rates of individual domains were nearly constant along a given orientation, as measured by the widths of the isotopically labeled bands. We plotted the growth rate as a function of temperature (Fig. 3G). According to the Arrhenius equation: $d r / d t \propto \exp \left(-E_{a} / k_{B} T\right)$, where $\bar{r}$ is the average radius (11), $E_{a}$ is the growth activation energy, $k_{B}$ is the Boltzmann constant, and $T$ is temperature. The value of $E_{a}$ was $1.76 \mathrm{eV}$ for graphene on $\mathrm{OF}-\mathrm{Cu}$ and 0.92 $\mathrm{eV}$ on $\mathrm{OF}-\mathrm{Cu}(\mathrm{O})$, indicating that, in the dynamic growth process, the barrier of the rate-limiting step is reduced.

For hydrocarbon (e.g., $\mathrm{CH}_{4}$ ) conversion to graphene on $\mathrm{Cu}$ during CVD growth, the following elementary steps are expected $(3,22,23)$ : (1) $\mathrm{CH}_{4}$ adsorption on $\mathrm{Cu}$ surfaces; (2) $\mathrm{CH}_{4}$ (partial-) dehydrogenation, resulting in carbon species such as $\mathrm{CH}_{x}(x=0-3)$; (3) surface diffusion of carbon species; and (4) carbon species attachment to graphene domain edge and incorporation into graphene lattice. The dehydrogenation of $\mathrm{CH}_{4}$ on $\mathrm{Cu}$ is endothermic (energetically unfavorable), and the diffusing carbon species on $\mathrm{Cu}$ are mainly $\mathrm{CH}_{x}(0<x<4)$, rather than atomic $\mathrm{C}$ (22). Density functional theory (DFT) calculations revealed that the hydrogen-terminated graphene edge on $\mathrm{Cu}$ is more energetically favorable than bare graphene edge on $\mathrm{Cu}$ (Fig. 3, $\mathrm{H}$ and I). Thus, $\mathrm{C}$ species edge attachment and lattice incorporation require dehydrogenation, e.g., $\mathrm{CH}_{x} \stackrel{\mathrm{Cu}}{\longrightarrow} \mathrm{CH}_{x-1}+\mathrm{H}(x=4,3,2,1)$, which is considered rate-limiting $(3,22,23)$. In contrast, theoretical studies have shown that pre-adsorbed oxygen on the $\mathrm{Cu}$ surface can enhance the dissociation of hydrocarbons (Fig. 3J) through the reaction: $\mathrm{CH}_{x}+\mathrm{O} \stackrel{\mathrm{Cu}}{\longrightarrow} \mathrm{CH}_{x-1}+\mathrm{OH}(x=4,3,2$, 1) $(24,25)$. Our DFT calculations have shown that the energy of $H$ in the form of $\mathrm{OH}$ group on $\mathrm{Cu}$ is lower than that of $\mathrm{H}$ on $\mathrm{Cu}$ by $0.6 \mathrm{eV} / \mathrm{H}$ (Fig. $3 \mathrm{I}$ ), pointing to a lower activation energy of edge dehydrogenation according to the Bell-Evans-Polanyi principle (26). Thus, experimental data from the isotope labeled growth and the atomic-scale calculations reveal that oxygen helps reduce the edge attachment barrier, facilitates $\mathrm{C}$ incorporation, and accelerates graphene growth. In the process, graphene growth proceeded by continuous carbon edge attachment and lattice incorporation, while surface oxygen species were consumed and then desorbed. This scenario does not necessarily conflict with the passivation effect of oxygen on graphene nucleation. The latter is governed by different kinetic processes (23) that typically require a much higher supersaturation density of carbon species than that during growth, in which dehydrogenation may not be the critical step.

The above model is supported by the change of graphene domain morphology with $\mathrm{O}_{2}$ exposure. Here, we focus on the domains on $\mathrm{Cu}$ (111). Figure 4A shows the typical shape of graphene domains formed on OF-Cu: compact hexagons with sharp edges, as obtained from the kinetic Wulff construction (27), which is expected as a result of edgeattachment-limited growth. In contrast, graphene domains grown on OF$\mathrm{Cu}(\mathrm{O})$ became multi-branched or dendritic (Fig. 4D), typical of diffusion (mass transport)-limited growth $(3,28)$. The morphology change indicates that, with the introduction of oxygen, carbon attachment at domain edges is no longer rate-limiting, and domain growth is instead governed by carbon diffusion or equivalently carbon flux, in agreement with our observations and analysis. The corresponding LEED patterns (Fig. 4, B and E) show only one set of hexagonal diffraction patterns, indicating that both domains are single crystals. Figure 4, C and F, sketch the relationship between the domain shapes and the graphene lattice. Both domains exhibit fast growth in the [2 $\left.\begin{array}{lll}2 & 1 & 0\end{array}\right]$ direction and slow growth in the [1 $\left.\begin{array}{lll}1 & 1 & 0\end{array}\right]$ direction, consistent with previous works, where hexagonal domains have been reported to have zigzag-terminated edges (29). Oxygen affected the domain shapes by modifying the growth kinetics but preserved the six-fold crystallographic symmetry. We further found that graphene domains on OF-Cu maintain a hexagonal shape when $P_{\mathrm{CH} 4}$ ranges from $1 \times 10^{-3}$ to $5 \times 10^{-2}$ Torr (fig. S9). However, on $\mathrm{OF}-\mathrm{Cu}(\mathrm{O})$ different domain shapes appear as a function of $P_{\mathrm{CH} 4}$ (Fig. 4, $\mathrm{G}$ to I). The sensitive dependence of domain morphology on carbon concentration again suggests that the growth kinetics has been brought into the diffusion-limited regime by oxygen.

To test the proposed growth mechanisms, a phase field model was developed to examine the domain shape evolution (11). Two key parameters, namely the characteristic attachment time of carbon species $\left(\tau_{\psi}\right)$ and carbon flux ( $F$, reflecting the $\left.P_{\mathrm{CH} 4}\right)$ are varied to simulate the experimental conditions. Note that the attachment time is closely related to the edge attachment barrier: the higher the energy barrier, the longer the characteristic attachment time. The symmetry of the graphene domains is dictated by the six-fold graphene edge energy, in agreement with experimental observations. The simulated domain shapes are shown in Fig. 4 , J to L, and fig. S9. When $\tau_{\psi}$ is long, the domain shape is hexagonal even if $F$ is changed within a large range; whereas when $\tau_{\psi}$ decreases, the hexagonal domain turns into six-branched at the same $F$. Furthermore, at the low $\tau_{\psi}$ value, $F$ becomes the dominant parameter and can tune the domain shapes from six-branched to dendritic. Thus, lowering the attachment barrier changes the domain shape in the same manner as $\mathrm{O}_{2}$ exposure in experiment. Thus, a rich variety of graphene domain morphologies $(30,31)$ can be explained and reproduced when the oxygen effect is considered in the growth kinetics. 


\section{References and Notes}

1. X. Li, W. Cai, J. An, S. Kim, J. Nah, D. Yang, R. Piner, A. Velamakanni, I. Jung, E. Tutuc, S. K. Banerjee, L. Colombo, R. S. Ruoff, Large-area synthesis of high-quality and uniform graphene films on copper foils. Science 324, 1312-1314 (2009). doi:10.1126/science.1171245 Medline

2. S. Bae, H. Kim, Y. Lee, X. Xu, J.-S. Park, Y. Zheng, J. Balakrishnan, T. Lei, H. Ri Kim, Y. I. Song, Y.-J. Kim, K. S. Kim, B. Özyilmaz, J.-H. Ahn, B. H. Hong, S. Iijima, Roll-to-roll production of 30-inch graphene films for transparent electrodes. Nat. Nanotechnol. 5, 574-578 (2010). doi:10.1038/nnano.2010.132 Medline

3. N. C. Bartelt, K. F. McCarty, Graphene growth on metal surfaces. MRS Bull. 37, 1158-1165 (2012). doi:10.1557/mrs.2012.237

4. A. W. Tsen, L. Brown, M. P. Levendorf, F. Ghahari, P. Y. Huang, R. W. Havener, C. S. Ruiz-Vargas, D. A. Muller, P. Kim, J. Park, Tailoring electrical transport across grain boundaries in polycrystalline graphene. Science 336, 1143-1146 (2012). doi:10.1126/science.1218948 Medline

5. P. M. Ajayan, B. I. Yakobson, Graphene: Pushing the boundaries. Nat. Mater. 10, 415-417 (2011). doi:10.1038/nmat3027 Medline

6. S. Bhaviripudi, X. Jia, M. S. Dresselhaus, J. Kong, Role of kinetic factors in chemical vapor deposition synthesis of uniform large area graphene using copper catalyst. Nano Lett. 10, 4128-4133 (2010). doi:10.1021/nl102355e Medline

7. I. Vlassiouk, M. Regmi, P. Fulvio, S. Dai, P. Datskos, G. Eres, S. Smirnov, Role of hydrogen in chemical vapor deposition growth of large single-crystal graphene. ACS Nano 5, 6069-6076 (2011). doi:10.1021/nn201978y Medline

8. Z. Yan, J. Lin, Z. Peng, Z. Sun, Y. Zhu, L. Li, C. Xiang, E. L. Samuel, C. Kittrell, J. M. Tour, Toward the synthesis of wafer-scale single-crystal graphene on copper foils. ACS Nano 6, 9110-9117 (2012). doi:10.1021/nn303352k Medline

9. H. Wang, G. Wang, P. Bao, S. Yang, W. Zhu, X. Xie, W. J. Zhang, Controllable synthesis of submillimeter single-crystal monolayer graphene domains on copper foils by suppressing nucleation. J. Am. Chem. Soc. 134, 3627-3630 (2012). doi:10.1021/ja2105976 Medline

10. Z. Zhang, M. G. Lagally, Atomistic processes in the early stages of thin-film growth. Science 276, 377-383 (1997). doi:10.1126/science.276.5311.377 Medline

11. Materials and methods are available as supplementary materials on Science Online.

12. X. Li, W. Cai, L. Colombo, R. S. Ruoff, Evolution of graphene growth on Ni and $\mathrm{Cu}$ by carbon isotope labeling. Nano Lett. 9, 4268-4272 (2009). doi: $10.1021 / \mathrm{n} 1902515 \mathrm{k}$ Medline

13. S. Nie, J. M. Wofford, N. C. Bartelt, O. D. Dubon, K. F. McCarty, Origin of the mosaicity in graphene grown on $\mathrm{Cu}(111)$. Phys. Rev. B 84, 155425 (2011). doi:10.1103/PhysRevB.84.155425

14. G. H. Han, F. Güneş, J. J. Bae, E. S. Kim, S. J. Chae, H. J. Shin, J. Y. Choi, D. Pribat, Y. H. Lee, Influence of copper morphology in forming nucleation seeds for graphene growth. Nano Lett. 11, 4144-4148 (2011). doi:10.1021/nl201980p Medline

15. J. Gao, J. Yip, J. Zhao, B. I. Yakobson, F. Ding, Graphene nucleation on transition metal surface: Structure transformation and role of the metal step edge. J. Am. Chem. Soc. 133, 5009-5015 (2011). doi:10.1021/ja110927p Medline

16. H. Chen, W. Zhu, Z. Zhang, Contrasting behavior of carbon nucleation in the initial stages of graphene epitaxial growth on stepped metal surfaces. Phys. Rev. Lett. 104, 186101 (2010). doi:10.1103/PhysRevLett.104.186101 Medline

17. B. Hammer, J. K. Nørskov, Theoretical surface science and catalysiscalculations and concepts. Adv. Catal. 45, 71-129 (2000). doi:10.1016/S0360 0564(02)45013-4

18. E. H. Hwang, S. Adam, S. D. Sarma, Carrier transport in two-dimensional graphene layers. Phys. Rev. Lett. 98, $186806 \quad$ (2007). doi:10.1103/PhysRevLett.98.186806 Medline

19. N. Petrone, C. R. Dean, I. Meric, A. M. van der Zande, P. Y. Huang, L. Wang, D. Muller, K. L. Shepard, J. Hone, Chemical vapor deposition-derived graphene with electrical performance of exfoliated graphene. Nano Lett. 12, 2751-2756 (2012). doi:10.1021/n1204481s Medline

20. C. R. Dean, A. F. Young, I. Meric, C. Lee, L. Wang, S. Sorgenfrei, K. Watanabe, T. Taniguchi, P. Kim, K. L. Shepard, J. Hone, Boron nitride substrates for high-quality graphene electronics. Nat. Nanotechnol. 5, 722 726 (2010). doi:10.1038/nnano.2010.172 Medline
21. E. Starodub, N. C. Bartelt, K. F. McCarty, Oxidation of graphene on metals. J. Phys. Chem. C 114, 5134-5140 (2010). doi:10.1021/jp912139e

22. W. Zhang, P. Wu, Z. Li, J. Yang, First-principles thermodynamics of graphene growth on Cu surfaces. J. Phys. Chem. C 115, 17782-17787 (2011). doi:10.1021/jp2006827

23. H. Kim, C. Mattevi, M. R. Calvo, J. C. Oberg, L. Artiglia, S. Agnoli, C. F. Hirjibehedin, M. Chhowalla, E. Saiz, Activation energy paths for graphene nucleation and growth on Cu. ACS Nano 6, 3614-3623 (2012). doi:10.1021/nn3008965 Medline

24. I. Alstrup, I. Chorkendorff, S. Ullmann, The interaction of $\mathrm{CH}_{4}$ at high temperatures with clean and oxygen precovered Cu(100). Surf. Sci. 264, 95102 (1992). doi:10.1016/0039-6028(92)90168-6

25. B. Xing, X. Y. Pang, G. C. Wang, C-H bond activation of methane on clean and oxygen pre-covered metals: A systematic theoretical study. J. Catal. 282 74-82 (2011). doi:10.1016/j.jcat.2011.05.027

26. R. A. van Santen, M. Neurock, S. G. Shetty, Reactivity theory of transitionmetal surfaces: A Brønsted-Evans-Polanyi linear activation energy-freeenergy analysis. Chem. Rev. 110, 2005-2048 (2010). doi:10.1021/cr9001808 Medline

27. V. I. Artyukhov, Y. Liu, B. I. Yakobson, Equilibrium at the edge and atomistic mechanisms of graphene growth. Proc. Natl. Acad. Sci. U.S.A. 109, 15136-15140 (2012). doi:10.1073/pnas.1207519109 Medline

28. T. A. Witten, Jr., L. M. Sander, Diffusion-limited aggregation, a kinetic critical phenomenon. Phys. Rev. Lett. 47, 1400-1403 (1981) doi:10.1103/PhysRevLett.47.1400

29. Q. Yu, L. A. Jauregui, W. Wu, R. Colby, J. Tian, Z. Su, H. Cao, Z. Liu, D. Pandey, D. Wei, T. F. Chung, P. Peng, N. P. Guisinger, E. A. Stach, J. Bao, S. S. Pei, Y. P. Chen, Control and characterization of individual grains and grain boundaries in graphene grown by chemical vapour deposition. Nat. Mater. 10, 443-449 (2011). doi:10.1038/nmat3010 Medline

30. B. Wu, D. Geng, Z. Xu, Y. Guo, L. Huang, Y. Xue, J. Chen, G. Yu, Y. Liu, Self-organized graphene crystal patterns. NPG Asia Materials 5, e36 (2013). doi:10.1038/am.2012.68

31. A. T. Murdock, A. Koos, T. B. Britton, L. Houben, T. Batten, T. Zhang, A. J. Wilkinson, R. E. Dunin-Borkowski, C. E. Lekka, N. Grobert, Controlling the orientation, edge geometry, and thickness of chemical vapor deposition graphene. ACS Nano 7, 1351-1359 (2013). doi:10.1021/nn3049297 Medline

32. J. W. Suk, A. Kitt, C. W. Magnuson, Y. Hao, S. Ahmed, J. An, A. K. Swan, B. B. Goldberg, R. S. Ruoff, Transfer of CVD-grown monolayer graphene onto arbitrary substrates. ACS Nano 5, 6916-6924 (2011). doi:10.1021/nn201207c Medline

33. S. Chen, H. Ji, H. Chou, Q. Li, H. Li, J. W. Suk, R. Piner, L. Liao, W. Cai, R. $\mathrm{S}$. Ruoff, Millimeter-size single-crystal graphene by suppressing evaporative loss of $\mathrm{Cu}$ during low pressure chemical vapor deposition. Adv. Mater. 25, 2062-2065 (2013). doi:10.1002/adma.201204000 Medline

34. Y. Hao, Y. Wang, L. Wang, Z. Ni, Z. Wang, R. Wang, C. K. Koo, Z. Shen, J. T. Thong, Probing layer number and stacking order of few-layer graphene by Raman spectroscopy. Small 6, 195-200 (2010). doi:10.1002/smll.200901173 Medline

35. R. Kobayashi, Modeling and numerical simulations of dendritic crystal growth. Physica D 63, 410-423 (1993). doi:10.1016/0167-2789(93)90120-P

36. L. Q. Chen, Phase-field models for microstructure evolution. Annu. Rev. Mater. Res. $\quad$ 32, 113-140 doi:10.1146/annurev.matsci.32.112001.132041

37. A. Karma, M. Plapp, Spiral surface growth without desorption. Phys. Rev. Lett. 81, 4444-4447 (1998). doi:10.1103/PhysRevLett.81.4444

38. www.fftw.org

39. G. Kresse, J. Furthmüller, Efficient iterative schemes for ab initio total-energy calculations using a plane-wave basis set. Phys. Rev. B 54, 11169-11186 (1996). doi:10.1103/PhysRevB.54.11169 Medline

40. G. Kresse, J. Hafner, Ab initio molecular dynamics for liquid metals. Phys Rev. B 47, 558-561 (1993). doi:10.1103/PhysRevB.47.558 Medline

41. G. Kresse, J. Hafner, Norm-conserving and ultrasoft pseudopotentials for first-row and transition elements. J. Phys. Condens. Matter 6, 8245-8257 (1994). doi:10.1088/0953-8984/6/40/015

42. D. Vanderbilt, Soft self-consistent pseudopotentials in a generalized eigenvalue formalism. Phys. Rev. B 41, 7892-7895 (1990). doi:10.1103/PhysRevB.41.7892 Medline

43. D. M. Ceperley, B. J. Alder, Ground state of the electron gas by a stochastic 
method. Phys. Rev. Lett. 45, 566-569 (1980). doi:10.1103/PhysRevLett.45.566

44. M. Vanin, J. J. Mortensen, A. K. Kelkkanen, J. M. Garcia-Lastra, K. S. Thygesen, K. W. Jacobsen, Graphene on metals: A van der Waals density functional study. Phys. Rev. B 81, 081408 (2010). doi:10.1103/PhysRevB.81.081408

45. H. J. Monkhorst, J. D. Pack, Special points for Brillouin-zone integrations. Phys. Rev. B 13, 5188-5192 (1976). doi:10.1103/PhysRevB.13.5188

Acknowledgments: We thank V. B. Shenoy (UPenn), Z. Zhang (USTC), Z. Li (USTC), N. C. Bartelt (Sandia), P. Sutter (Brookhaven), G. Wang (Nankai Univ.), C. Gong (UT-Dallas), Z. Yan (Texas A\&M), and C. R. Dean (City College of New York) for valuable discussions. We thank K. Watanabe and $\mathrm{T}$. Taniguchi for providing h-BN substrates. This work acknowledges support from the W. M. Keck Foundation, the Office of Naval Research (ONR) and SWAN NRI. Work at Columbia was supported by the Center for Re-Defining Photovoltaic Efficiency through Molecular-Scale Control, an Energy Frontier Research Center funded by the U.S. Department of Energy (DOE), Office of Science, Office of Basic Energy Sciences under Award DE-SC0001085, National Science Foundation (NSF) DMR - 1124894, and ONRN000141310662. Work at IHPC was supported by the Agency for Science, Technology And Research (A*STAR), Singapore. Work at Sandia was supported by the Office of Basic Energy Sciences, Division of Materials and Engineering Sciences, U.S. DOE under contract no. DE-AC04-94AL85000. Work at Rice was supported by the ONR MURI and NSF (CBET). The firstprinciples computations were performed on NICS Kraken (NSF OCI1053575), NERSC Hopper (DOE DE-AC02-05CH11231), and DAVINCI (NSF OCI-0959097). H.C. acknowledges support from NSF DMR-1122603. A relevant patent application is in process.

\section{Supplementary Materials}

www.sciencemag.org/cgi/content/full/science.1243879/DC1

Materials and Methods

Supplementary Text

Figs. S1 to S12

References (32-45)

29 July 2013; accepted 1 October 2013

Published online 24 October 2013; 10.1126/science.1243879 

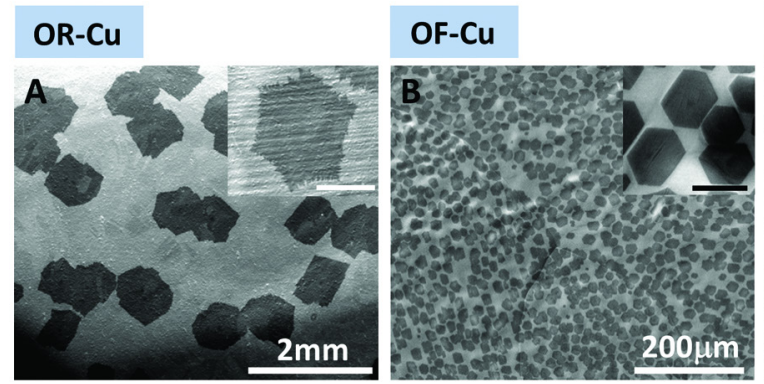

\section{$\mathrm{OF}-\mathrm{Cu}(\mathrm{O})$}

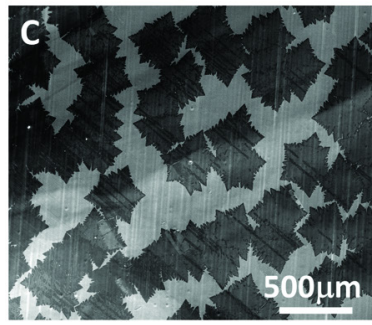

Fig. 1. The effect of oxygen on graphene nucleation density and domain shapes on Cu. SEM (SEM) images of graphene domains grown on (A) OR-Cu, (B) OF-Cu, and (C) OF-Cu (O), respectively. In all cases, the $P_{\mathrm{CH} 4}=1 \times 10^{-3}$ Torr, and $P_{\mathrm{H} 2}=0.1$ Torr, the growth time was $150 \mathrm{~min}$ for $(\mathrm{A})$ and $(\mathrm{C})$ and $50 \mathrm{~min}$ in $(\mathrm{B})$. The insets in $(\mathrm{A})$ and $(\mathrm{B})$ are the high magnification SEM images in each case. The scale bar is $500 \mu \mathrm{m}$ in the inset of (A), and $20 \mu \mathrm{m}$ in the inset of (B).
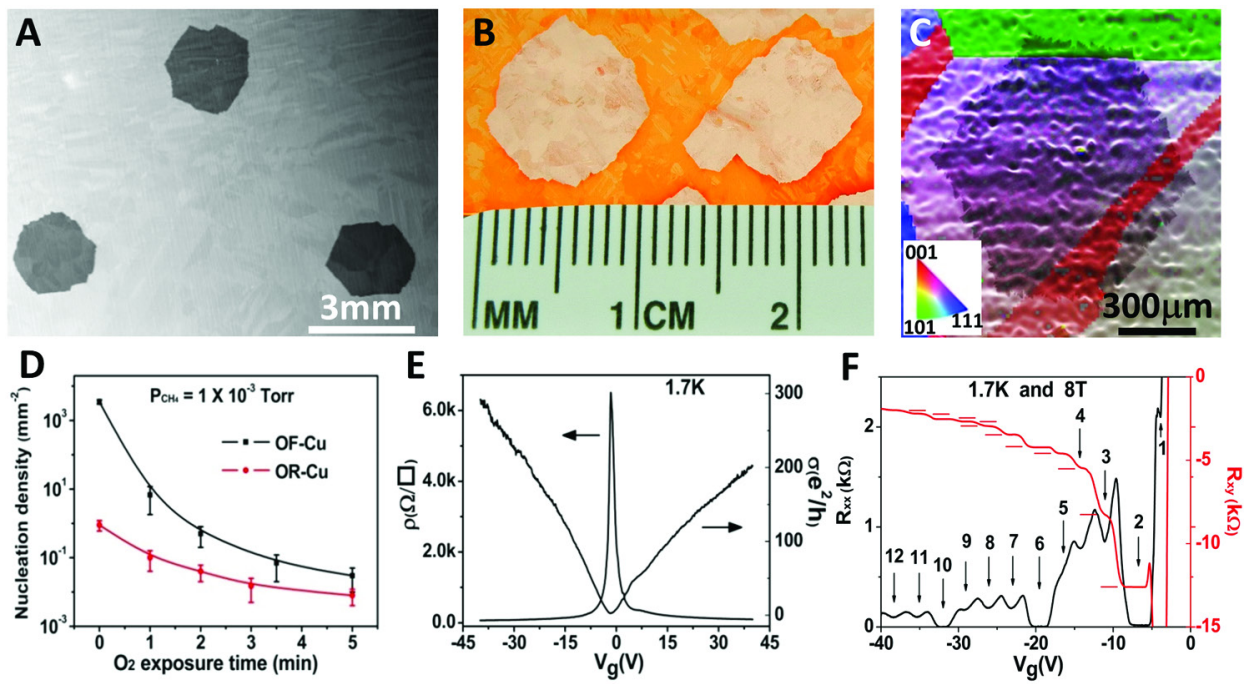

Fig. 2. Size, structure, and electrical transport properties of large graphene domains grown on $\mathrm{Cu}$ exposed to $\mathrm{O}_{2 .}$ (A) SEM image of low density graphene domains on OR-Cu exposed to $\mathrm{O}_{2}$. (B) Optical image of centimeter-scale graphene domains on OR-Cu exposed to $\mathrm{O}_{2}$. The sample was prepared by heating in air at $180^{\circ} \mathrm{C}$ for 30 min to oxidize bare $\mathrm{Cu}$ (orange) and visualize graphene domains (pale), as reported by Wang et al. (9). (C) The graphene nucleation density as a function of $\mathrm{O}_{2}$ exposure time. (D) Superimposed SEM and EBSD images of a graphene domain grown across Cu multi-grains. (E) Plots of resistivity and conductivity as a function of gate voltage at $1.7 \mathrm{~K}$. $(\mathbf{F})$ Longitudinal resistivity, $R_{\mathrm{xx}}$, measured on left axis (black) and Hall resistance, $R_{\mathrm{xy}}$, on right axis (red) as a function of $V_{\mathrm{g}}$. 

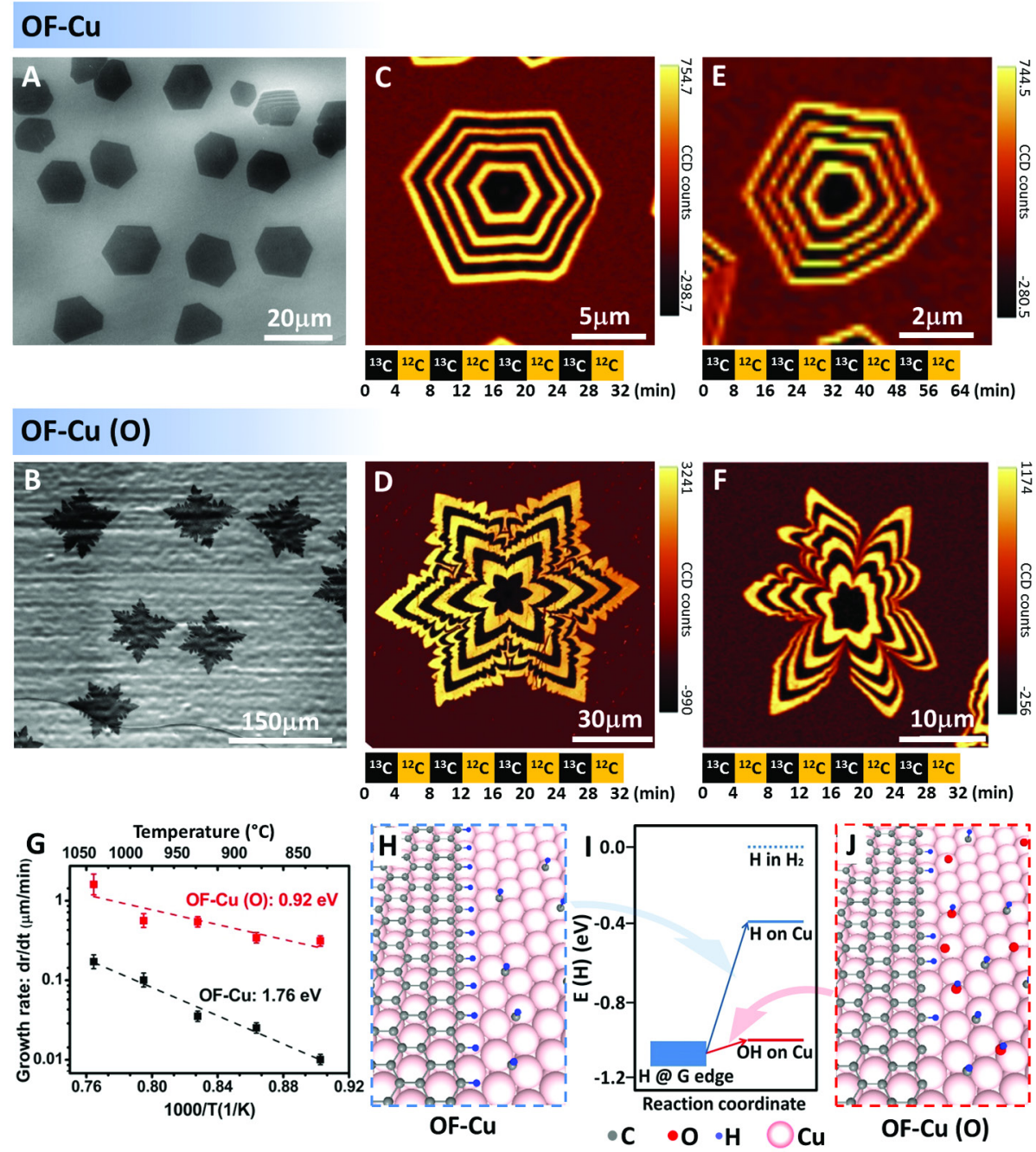

Fig. 3. The effect of oxygen on graphene growth kinetics. SEM images of graphene domains grown on (A) OF-Cu, and (B) OF-Cu (O). Isotope-labeled Raman maps of the 2D (G') band intensities on Si substrates for growth at (C and D) $1035^{\circ} \mathrm{C}$ and $(\mathbf{E}$ and $\mathbf{F}) 885^{\circ} \mathrm{C}$. The isotope switching intervals are indicated below each image. (G) Logarithmic plots of graphene domain growth rate $d \bar{r} / d t v s 1 / \mathrm{T}$. The error bars are from calculations of different domains for each case, and the activation energy $E_{a}$ is extracted from the slope of the linear fit. $(\mathbf{J}$ and $\mathbf{H})$ Atomic-scale schematics of graphene edge growth on Cu with and without the assistance of oxygen, respectively. (I) DFT calculated energies of different configurations of $\mathrm{H}$ attachment, in reference to $\mathrm{H}$ in $\mathrm{H}_{2}$. The energy spread of $\mathrm{H}$ at the graphene edge is due to the computational uncertainty resulting from the lattice mismatch between graphene and $\mathrm{Cu}$. 


\section{OF-Cu}

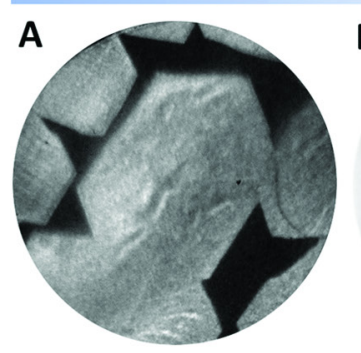

B

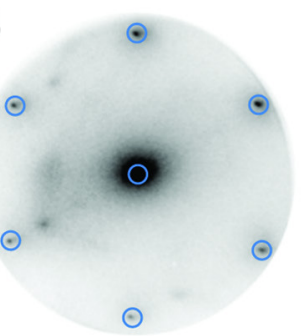

○

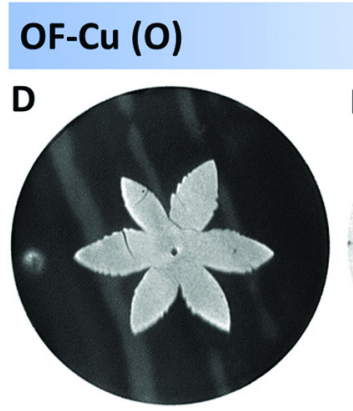

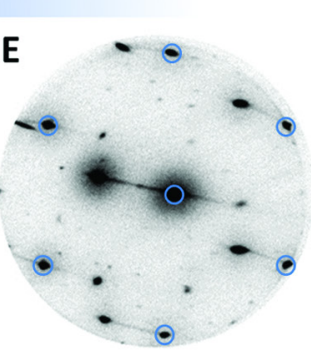

○

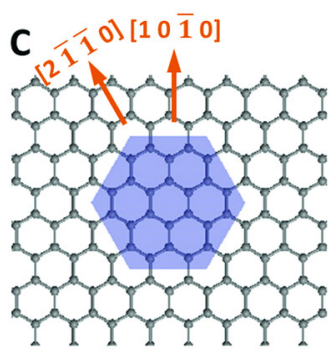

\section{$\mathrm{OF}-\mathrm{Cu}(\mathrm{O})$}

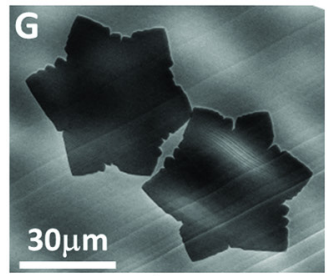

$P_{\mathrm{CH} 4}=1 \times 10^{-2}$ Torr
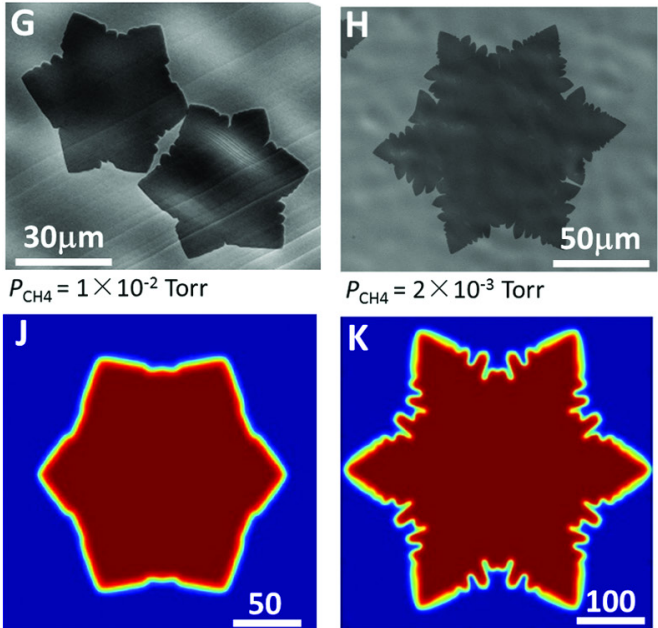

$F=0.02 ; \tau_{\psi}=1.0$

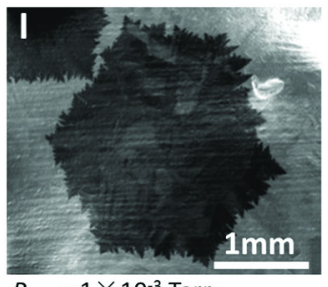

$P_{\mathrm{CH} 4}=1 \times 10^{-3}$ Torr

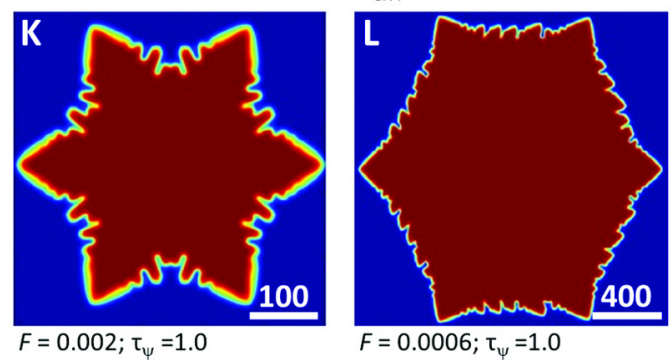

Fig. 4. The effect of oxygen on graphene domain shapes on $\mathbf{C u}$. (A, B, D, and E) LEEM images and corresponding LEED patterns (blue circles) of graphene domains on OF-Cu and OF-Cu (O), respectively. The extra LEED spots came from the faceted $\mathrm{Cu}$. The viewing fields in (A) and (D) are 20 and $60 \mu \mathrm{m}$, respectively. (C and F) Schematics of growth directions of the two types of graphene domains. ( $G$ to I) SEM images of graphene domains grown on OF-Cu (O) as a function of $P_{\mathrm{CH} 4}$. ( $\mathbf{J}$ to $\mathbf{L}$ ) Phase field simulation results as a function of characteristic attachment time and carbon flux. The scale bars in (J) to (L) refer to simulation units, corresponding to the lengths. 